\title{
Sens du juste et usages du droit du travail : une évolution contrastée entre la France et la Grande- Bretagne au XIX siècle
}

The meaning of being fair and the uses of labour law: a contrasted evolution in 19th-century France and Britain

\section{Alain Cottereau}

\section{CpenEdition}

\section{Journals}

Édition électronique

URL : http://journals.openedition.org/rh19/1148

DOI : 10.4000/rh19.1148

ISSN : $1777-5329$

Éditeur

La Société de 1848

\section{Édition imprimée}

Date de publication : 1 décembre 2006

Pagination : 101-120

ISSN : 1265-1354

Référence électronique

Alain Cottereau, «Sens du juste et usages du droit du travail : une évolution contrastée entre la France et la Grande-Bretagne au xix ${ }^{\mathrm{e}}$ siècle ", Revue d'histoire du XIXe siècle [En ligne], 33 | 2006, mis en ligne le 01 décembre 2008, consulté le 21 décembre 2020. URL : http://journals.openedition.org/rh19/1148 : DOI : https://doi.org/10.4000/rh19.1148 


\section{ALAIN COTTEREAU}

\section{Sens du juste et usages du droit du travail: une évolution contrastée entre la France et la Grande-Bretagne au XIXe siècle ${ }^{1}$}

Les tribunaux des prud'hommes sont une institution française très mal connue, non seulement dans l'histoire sociale comparative internationale, mais même dans l'histoire sociale française. Bien des raisons expliquent ce désintérêt, à commencer par les rejets idéologiques les plus divers. D'un côté, le mépris du droit par les anciens courants ouvriéristes et marxistes français a rejeté dans l'oubli un dispositif de relations sociales axé sur le consensus de justice et la conciliation. De l'autre côté, les courants de libéralisme économique évitaient de s'intéresser à une institution de régulation des relations sociales, dont le succès ne rentrait pas dans le cadre du laissez-faire. Les traditions de paternalisme ne s'y intéressaient pas pour autant, car les notables médiateurs étaient exclus par la désignation élective directe des prud'hommes patrons et ouvriers. Mais les raisons les plus décisives de la méconnaissance des prud'hommes ne sont pas externes. Elles sont liées aux ressorts mêmes de l'institution, dès l'origine. En effet, durant le XIX ${ }^{e}$ siècle, le fonctionnement et le succès des prud'hommes impliquaient l'occupation d'une place très originale, à distance des institutions publiques et des arrangements privés : le dispositif, centré sur la conciliation individuelle des conflits, mettait en œuvre les opinions de milieux professionnels, patrons et ouvriers, et les appelait à élaborer un consensus collectif tout en évitant les formes de publicité et de représentation collective ouverte. Suivant cette logique originale de publicité limitée, une sorte de droit coutumier, jamais formulé en principes, géré au jour le jour, a été instauré et maintenu en marge, et parfois en contradiction avec le système dominant de droit français. Ainsi, le confinement hors espace public national faisait partie du système de droit prud'homal, ce qui contribue à expliquer l'état de méconnaissance où il a été maintenu jusqu’à une période récente.

L’originalité du statut de ce droit des ouvriers, régulé par les prud'hom-

1. Texte publié en anglais sous le titre : «Industrials tribunals and the establishment of a kind of common law of labour in nineteenth-century France», dans Willibald Steinmetz (ed.), Private Law and Social Inequality in the Industrial Age: Comparing Legal Cultures in Britain, France, Germany and the United States, Oxford, Oxford University Press, 2000, p. 203-226. 
mes, a amené à évoquer dans le titre une sorte de common law. Le terme est provocateur et ne doit évidemment pas être pris trop littéralement. Mais il attire l'attention sur certains traits de l'établissement de ce droit des ouvriers, très éloigné du «légicentrisme» instauré en France depuis la Révolution française, et plus proche d'un droit typiquement britannique aux yeux des continentaux : un droit constitué essentiellement par les décisions judiciaires, de type casuistique, se rationalisant à l'aide de précédents, et invoquant davantage le sens commun et le sens de la justice que l'exégèse des textes statutaires pour justifier son activité législatrice.

Dans la présente étude, on donnera un aperçu du contenu et du fonctionnement de ce droit en établissant une comparaison avec le droit britannique de l'emploi ouvrier, durant les mêmes époques (les deux premiers tiers du $\mathrm{XIX}^{\mathrm{e}}$ siècle). Cette étude s'appuie, du côté français, sur des enquêtes menées depuis une quinzaine d'années parmi des archives prud'homales, et quelques autres archives, souvent inconnues ou non classées au moment de leur étude. Il existe très peu de publications appuyées sur l'examen des décisions prud'homales effectives ${ }^{2}$. Par contre, pour la comparaison avec le cas britannique, on s'est limité à des emprunts à l'histoire sociale et à des enquêtes rapportées par les papiers parlementaires.

2. Lorsque l'étude ne renverra pas à une source ou à une publication particulière, elle s'appuiera sur ces enquêtes en archives prud'homales, dont voici les principales publications : un aperçu général de l'institution prud'homale et de l'enquête est donné dans mon article : "Justice et injustice ordinaire sur les lieux de travail, d'après les audiences prud'homales, 1806-1866», dans Le Mouvement social, $\mathrm{n}^{\circ} 141$, octobre-décembre 1987 , p. 25-61. Depuis lors la recherche s'est étendue à une quinzaine de conseil prud'homaux et a mis au jour de nouvelles archives, mais sans rien changer aux grandes lignes exposées en 1987. Le numéro spécial du Mouvement social comporte deux autres articles fondés sur des enquêtes de première main (Paul Delsalle, «Tisserands et fabricants chez les prud'hommes dans la région de Lille-Roubaix-Tourcoing (1810-1848)», p. 61-80; Heinz-Gerhard Haupt, "Les employés lyonnais devant le Conseil de prud'hommes du commerce (1910-1914)», p. 81-100), ainsi que des articles de juristes fondés sur des sources juridiques secondaires. Ma contribution : « «Esprit public» et capacité de juger", dans Alain Cottereau et Paul Ladrière [dir.], Pouvoir et légitimité - Figures de l'espace public, Paris, ÉHÉSS, p. 239-273, comporte aussi des éclairages sur les prud'hommes. Une publication plus spécialisée : Alain Cottereau, "L’embauche et la vie normative des métiers durant les deux premiers tiers du XIX ${ }^{e}$ siècle français", dans Les Cahiers des relations professionnelles, ${ }^{\circ} 10$, février 1995 , p. 47 71 , étudie un segment de droit des ouvriers régulé par les prud'hommes, le «marchandage». Un essai publié en anglais : Alain Cottereau, "The fate of "Fabriques collectives" in the industrial world : the examples of the silk industries in Lyons and London, 1800-1850", dans Charles F Sabel et Jonathan Zeitlin (eds), World of Possibilities. Flexibility and Mass Production in Western Industrialisation, Cambridge, Cambridge University Press, 1997, p. 75-153, détaille la régulation économique exercée par les prud'hommes lyonnais, en comparaison avec la dérégulation exercée à la même époque dans les soieries londoniennes. Mon article : «Droit et bon droit. Un droit des ouvriers instauré puis évincé par le droit du travail (France, XIX ${ }^{\mathrm{e}}$ siècle)", dans Annales. Histoire, sciences sociales, n 6, novembre-décembre 2002, p. 1521-1557, expose le fonctionnement et l'évolution du droit des ouvriers instauré par les prud'hommes au XIX ${ }^{e}$ siècle, analyse son oubli historiographique, et le situe dans l'histoire du sens de la justice au sein de la société française. 


\section{Masters and Servants. Les évolutions CONTRAIRES DU CAS FRANÇAIS ET BRITANNIQUE.}

Le rapprochement entre les jurisprudences, dans le domaine du travail, fait ressortir un contraste majeur : tandis qu'en Grande-Bretagne, les lois statutaires de l'emploi resserrent l'assimilation des ouvriers à des serviteurs, jusqu'aux réformes législatives de 1866-1875, la France connait une évolution exactement contraire : à partir de 1789, la jurisprudence consacre solennellement une stricte séparation entre d'un côté les relations d'emploi ouvrier, le «louage d'ouvrage", de l'autre les relations de service domestiques, le «louage de service». Par la suite, une inversion jurisprudentielle et doctrinale intervient, à partir de 1866, qui tend au contraire à assimiler le «louage» des ouvriers au "louage de services", au moment même où en Grande-Bretagne commençaient à être remises en cause les Laws of Masters and Servants (employeurs et ouvriers). L'évolution est bien connue pour le cas de la Grande-Bretagne, elle l'est beaucoup moins pour le cas de la France, aussi allons-nous en résumer les grands traits.

\section{La Révolution française et la liberté des ouvriers}

En 1789, l'état de la jurisprudence française était marqué par une ambiguïté conflictuelle omniprésente : les ouvriers salariés, c'est-à-dire les ouvriers, artisans ou artistes "pour compte d'autrui " étaient traités tantôt comme "locateurs d'ouvrage», en positions de réciprocité marchande légitime avec leurs employeurs, tantôt comme des serviteurs, soumis à des obligations de subordination, ce qui excluait la légitimité du marchandage réciproque ${ }^{3}$. Les historiens du Second Empire et de la Troisième République, suivis par les historiens du droit du travail, ont surtout insisté sur les obligations juridiques de subordination, impliquées par les décisions réglementaires du roi et des cours de justices ${ }^{4}$. Mais une recherche récente ${ }^{5}$, la première qui ait regardé de près les débats judiciaires du XviII ${ }^{\mathrm{e}}$ siècle sur le travail, et qui ait pris en compte les points de vue exprimés des justiciables, a montré l'extrême instabilité et conflictualité des normes mises en avant, tout au long du XvIII ${ }^{e}$ siècle. Dans cette diversité on peut retenir, entre autres, un affrontement constant entre d'un côté, la légitimité de liberté des ouvriers, revendiquée par les compagnons, et soutenue épisodiquement par une partie des ouvriers et des maîtres, de l'autre côté, l'impératif de soumission du «service», que voulaient imposer périodiquement des initiatives de maîtres coalisés, soutenus le plus souvent

3. Steven L. Kaplan, "Réflexions sur la police du monde du travail», dans Revue historique, t. CCLXI, 1979, p. 17-77.

4. Emile Levasseur, Histoire des classes ouvrières en France depuis la conquête de Jules César jusqu’à la Révolution, Paris, Guillaumin, 1859.

5. Michael Sonenscher, Work and Wages. Natural Law, Politics and the Eighteenth-Century French Trades, Cambridge, Cambridge University Press, 1989. 
par la haute hiérarchie judiciaire et administrative, tandis que les magistrats locaux étaient davantage portés à transiger ou à reconnaître une légitimité aux demandes ouvrières.

Cette légitimité, couramment incorporée dans les pratiques de négociation sur les lieux de travail, trouvait un cadre d'expression dans l'invocation du droit naturel contre le droit positif : "Nous ne sommes pas des esclaves» était un leitmotiv. L'expression faisait référence à l'ancienne identité latine des termes de serviteur et d'esclave, par delà les différenciations séculaires de statut instaurées entre esclavage, servitudes volontaires et domesticité. L'invocation du droit naturel était moins l'appel d'un contenu doctrinal précis qu'un acte d'autonomie du jugement, manifestant une compétence pour critiquer le bien fondé du droit positif, en vertu de principes supérieurs, communs à l'humanité. Ces actes critiques invoquaient aussi le "droit commun" ou le «droit des gens», sans toujours reprendre leur signification technique juridique, mais en leur donnant à peu près la même signification de recours critique aux principes communs de l'humanité.

Cet arrière-plan historique est décisif pour comprendre la puissance émancipatrice de la Révolution française, dans le domaine du droit du travail. La proclamation des droits de l'homme, les principes de liberté politique et civile, l'abolition de toutes les instances de régulation corporative, les fameuses lois Le Chapelier ne consacrèrent pas seulement des «libertés économiques" chères aux économistes classiques, et tout aussi chères aux anciens schématismes de la «Révolution bourgeoise». Ces bouleversements proclamés furent vécus intensément comme une émancipation ouvrière effective, comme un triomphe des anciennes luttes morales, et comme la consécration d'une capacité effective de négocier équitablement avec les employeurs. Il ne s'agissait pas seulement de nouveaux droits civils formels, mais bien de nouvelles possibilités réelles, massivement utilisées. Ce que le langage plus courant de l'époque appelait une «révolution des mœurs».

L'aspect émancipateur du salariat ouvrier ne s'est guère traduit dans des textes ni dans des listes de nouveaux droits. Pour l'apprécier, il faut surtout suivre localement les jurisprudences avant et après 1789 , et les situer dans les relations industrielles effectives. À ce moment-là, la table rase des anciennes régulations statutaires prend une signification très précise, dans chaque histoire locale. Dans le cas des soieries lyonnaises par exemple, l'abolition des anciens règlements et de leurs anciennes juridictions débouche sur un nouveau régime des relations entre négociants, ouvriers-chefs d'atelier, compagnons (salariés des ouvriers-chefs d'atelier) et apprentis. Dans tous les cas, la levée des entraves réglementaires aux départs et aux changements d'employeurs est utilisée comme une possibilité nouvelle de marchander équitablement les conditions de travail et de rémunération : les délais d'avertissement avant départ deviennent strictement réciproques, et la menace permanente de quitter pour des conditions plus favorables de rémunération permet aux 
chefs d'atelier et aux compagnons de maintenir un alignement sur les cours de «façon" (tarifs de main-d'œuvre, officieux ou officiels selon les époques) les plus favorables ${ }^{6}$.

Entre mille attestations de cette révolution des mœurs, une série de traces remarquables est constituée par les rapports et pétitions de patrons entre 1794 et 1804, lorsque après la Terreur, il fallut construire ou reconstruire un ordre industriel à partir des nouveaux principes. Les multiples lettres, pétitions, mémoires envoyés par les manufacturiers et leurs associations aux administrations locales ou nationales constatent tous un nouveau climat des relations de travail, pour le déplorer ou l'admettre : l'esprit de liberté qui a soufflé sur tous les rapports sociaux s'est imposé sur les lieux du travail, et rend impossible le retour aux anciennes règles de soumission. Les descriptions les plus vives viennent des manufacturiers qui demandent un retour aux anciennes règles du droit du travail. Dans ce cas, ils dressent un tableau désastreux de leur impuissance, face à «l'esprit de liberté» des ouvriers et réclament en conséquence l'appui de l'autorité pour rétablir d'anciens règlements, d'anciens usages, et, avec eux, "restaurer la subordination». Des pétitions patronales expliquent même qu'il est dangereux pour l'intégrité physique d'un manufacturier de seulement évoquer les anciennes coutumes d'embauche et de renvoi ${ }^{7}$

Les arguments et anecdotes rapportés par toutes ces pétitions établissent un contraste entre les anciennes limitations de la liberté de départ des ouvriers et la situation nouvelle instaurée depuis 1790 . Sous divers langages d'approbation ou de désapprobation, ils explicitent comment d'anciennes jurisprudences de contrôle, chargées de maintenir l'obéissance, restreignaient la négociation interindividuelle, tandis que le nouveau régime donnait au contraire libre cours à la discussion sur un pied d'égalité. Certains exposent ainsi comment les ouvriers se livrent à des "chantages», c'est-à-dire comment ils menacent de quitter leurs employeurs si ceux-ci ne réalignent pas leurs conditions de travail et de salaire sur tel ou tel patron concurrent, plus favorable aux ouvriers. Cependant d'autres patrons, et avec eux les tribunaux de commerce et instances consultatives (bureaux consultatifs ayant remplacé

\footnotetext{
6. Une analyse plus détaillée de ces changements avant et après la Révolution à Lyon est donnée dans ma contribution : "The fate of "Fabriques collectives»... ", art. cité.

7. Par exemple une pétition des manufacturiers tisseurs de Rouen (juillet 1804, Archives départementales de Seine-Maritime, $10 \mathrm{M}$ 4), évoque l'esprit de liberté des ouvriers, leurs habitudes de changer d'employeur dès que les conditions ou le prix ne leur conviennent pas, au nom des nouveaux principes de liberté, à l'encontre des anciens règlements. Les pétitionnaires réclamaient un retour à ces anciens règlements, aux restrictions du droit de quitter, à leurs sanctions pénales. Ils n'obtiendront pas satisfaction, pas plus que les nombreux pétitionnaires à travers la France. Toutes ces demandes de restriction au libre changement d'employeur se verront opposer par les gouvernements successifs du Directoire, du Consulat et de l'Empire, les nouveaux principes intangibles de liberté, impliquant la réciprocité de négociation et de sanction. De multiples dossiers de cette sorte se trouvent dans les archives locales, départementales et nationales, chronologiques ou classés dans les affaires commerciales et judiciaires. D'autres se trouvent disséminés dans les dossiers par branches des Archives Nationales, série F 12 pour les périodes concernées.
} 
les Chambres de Commerce pendant la Révolution) soutiennent majoritairement que ces soi-disant "chantages" ouvriers ne doivent pas être considérés comme des insubordinations, mais bien comme l'exercice légitime de la nouvelle liberté. Les autorités ministérielles et policières adoptent cette position. Les rares exceptions, notamment policières, qui répriment des comportements d'insubordination sur dénonciation patronale, dans l'esprit de surveillance paternaliste d'avant la Révolution, sont toujours désavouées par la hiérarchie judiciaire et administrative.

\section{L'exemple du "livret ouvrier» et de la liberté de quitter}

Une bonne illustration de ce nouveau climat de liberté ouvrière est constituée par les débats sur le rétablissement du «livret ouvrier» puis par la jurisprudence de sa mise en application. Ce qu'on appelle en France le «livret ouvrier » est un document issu de multiples règles statutaires et jurisprudentielles d'Ancien Régime : dans la plupart des localités manufacturières, nul ouvrier ne pouvait quitter un maître sans disposer d'un «billet de congé» du maitre, attestant qu'il avait rempli ses engagements et n'avait pas de dette. Les divers règlements statutaires locaux modulaient cette règle et précisaient les délais d'avertissement nécessaires avant le congé (délais souvent imposés aux seuls ouvriers, ou encore d'une durée plus longue pour les ouvriers que pour les maitres). Dans bien des cas, ils prescrivaient que le "billet de congé» devait expliquer le motif du départ de l'ouvrier, ou même évaluer sa conduite et ses «mœurs" pour en informer l'employeur suivant ${ }^{8}$. Un débat eut lieu en France entre 1794 et 1803 pour savoir si on rétablirait ces règles. Or il est remarquable qu'il y eut un large consensus entre non seulement les ouvriers consultés, mais aussi entre patrons et entre administrations, pour ne pas restaurer ces règles.

À la place fut établie, en 1803-1804, une législation qui prévoyait un "livret», (c'est-à-dire un petit carnet unique des différents "billets de congé») sur des bases très différentes. Tout d'abord, elle en faisait, non un document de surveillance déléguée par l'administration aux employeurs, mais une pièce de droit conventionnel privé, destinée à attester certains aspects du contrat de travail, tout comme le droit commercial prescrivait certaines écritures de livres et de transactions : certifier un engagement d'ouvrage et son achèvement ("quittance») ou encore reconnaître que des avances de salaires restent

8. Voici à titre d'illustration un article des «Règlements et statuts...» des manufactures de drap d'Elbeuf, du 19 avril 1667, comportant l'obligation de l'enquête morale et la dissymétrie du délai d'avertissement avant de quitter : "XXIV. Un tisserand ne pourra quitter son maître, que la pièce qu'il aura montée sur le métier ne soit achevée, \& à ce sujet sera tenu d'avertir son maître en montant la pièce. Et si l'ouvrier doit quelque chose à son maître, celui chez lequel il ira travailler, sera obligé de s'informer des vies \& mœurs dudit ouvrier, \& du sujet pour lequel il a quitté ledit maître, lequel sera tenu payer ce que ledit ouvrier devra au maître qu'il aura quitté, comme aussi pour ce qu'il devra des fautes qu'il aura fait à sa besogne. Le maître aussi ne pourra congédier un ouvrier, qu'il ne l'ait averti 24 heures auparavant." D'autres statuts explicitent la "contrainte par corps» pour renvoyer de force chez leurs maitres les ouvriers qui auraient quitté sans congé ou sans "cause légitime». 
dues, à solder par le futur employeur, selon des limites compatibles avec la faible solvabilité présumée de l'ouvrier. Le caractère "civil» sur lequel insistent les législateurs et la jurisprudence signifie que le livret est détaché de toute considération d'ordre public. Ce caractère se traduit avant tout dans le fait qu'il n'y a plus aucun dispositif répressif, contrairement aux réglementations du billet de congé sous l'Ancien Régime. Désormais il n'y a ni prison, ni amende, ni obligation de retour chez l'employeur, en cas de nonrespect de la législation des livrets. Les manquements aux obligations du livret ne sont susceptibles que de dommages et intérêts, au profit de la partie lésée, patron ou ouvrier. Les jurisprudences prud'homales montrent d'ailleurs que des dommages et intérêts sont versés au moins aussi fréquemment à des ouvriers qu'à des employeurs, au début de la mise en pratique de ces règles : les ouvriers qui veulent quitter, par exemple pour de meilleurs salaires, et à qui le livret est refusé par l'employeur sans motif suffisamment sérieux et prouvé, obtiennent couramment aux prud'hommes la restitution de leur livret, accompagnée d'une indemnité pour le temps et le salaire perdus ${ }^{9}$.

En second lieu, les règles puis les jurisprudences de l'inscription des dettes fonctionnent effectivement selon l'esprit des promoteurs du nouveau droit civil : il s'agit, non de mesures de restriction aux changements d'emploi, mais au contraire de mesures facilitant la mobilité. L'inscription des dettes sur le livret est un dispositif qui rend possible le changement d'employeur avant de solder les avances reçues pour salaire, outillage ou équipement d'atelier ${ }^{10}$. Tandis que le droit commun permettrait à l'employeur de retenir l'ouvrier endetté par pression économique, la jurisprudence sur les livrets permet de faire circuler les dettes d'employeur à employeur : lorsqu'un ouvrier endetté

9. Pour des indications supplémentaires sur les livrets, voir Alain Cottereau, «Justice et injustice ordinaire...", art. cité (un exemple détaillé de jugement avec restitution de livret à une ouvrière d'usine, avec dommages et intérêt, y est exposé p. 26-29), et "The fate of "Fabriques collectives"...", art. cité. Le fonctionnement libéral de la législation du livret est d'autant plus nécessaire à souligner qu'il a donné lieu aux historiographies les plus fantaisistes à partir de la Troisième République : des juristes ultra-individualistes et anti-réformistes (notamment Marc Sauzet, "Le livret obligatoire des ouvriers ", dans Revue critique de droit et de jurisprudence, 1890, p. 21-30 et "Essai historique sur la législation industrielle de la France", dans Revue d'économie politique, 1892, p. 353-356, 890-930, 1097-1136) voulurent démontrer, à partir des années 1880, qu'il y avait eu continuité d' "interventionnisme» et d'arbitraire policier entre les législations pré- et post-révolutionnaire, notamment en matière de droit du travail et du livret. Ses écrits multiplièrent les affirmations fausses et infondées en matière de documentation historiographique et juridique. Par exemple, il soutint la thèse d'une continuité entre les billets de congé d'Ancien Régime et le livret institué en 1803, en jouant sur des significations anachroniques de la vieille notion française de "police» et en raisonnant comme si les sanctions pénales n’avaient pas été supprimées irrévocablement sous la Révolution. Ses affirmations péremptoires masquaient une ignorance complète des jurisprudences locales. Cet auteur n'aurait pas à être mentionné s'il n'avait malheureusement été souvent répété sans examen critique, dans les traditions académiques françaises d'histoire du droit. Du coup, des auteurs anglais qui leur ont fait confiance ont été induits en erreur sur des points comparatifs essentiels : ils ont cru pouvoir établir une ressemblance entre le droit anglais de l'emploi et les supposées restrictions françaises à la liberté de quitter, durant les années 1800-1870.

10. Les textes créent une obligation aux employeurs de laisser partir les ouvriers endettés lorsque l'initiative de cessation ou de modification du louage d'ouvrage vient du patron. Par contre, en droit, le patron peut exiger le remboursement préalable, lorsque la cessation ou la modification est demandée par l'ouvrier. Cependant la plupart des jurisprudences prud'homales établissent une règle de liberté de quitter avant remboursement sans chercher à savoir d'où vient l'initiative. 
veut changer de patron, qu'il soit à domicile ou en usine, il peut demander l'«inscription» de sa dette. Cela signifie que son futur employeur sera responsable du remboursement auprès de l'employeur précédent, par prélèvement sur le futur salaire dans une limite de 1/8. L'ouvrier y améliore sa force de négociation, et le patron y trouve une garantie relative de remboursement, lui permettant d'ouvrir un crédit au recrutement, et de financer les frais d'outillage ou d'équipement des ateliers d'ouvriers.

Dès lors, le livret fonctionne comme une sorte de registre de billets d'escompte sur le travail, et fournit l'instrument d'un dispositif de crédit gratuit aux ouvriers et à leurs ateliers : il donne aux détenteurs la possibilité d'obtenir des avances gratuites, garanties par leur seul travail futur chez leurs employeurs futurs successifs, sur la foi de leur livret et sous la caution éventuelle des prud'hommes. En cas de non-remboursement, il n'y a pas de sanctions pénales ni de contraintes punitives, contrairement aux sanctions $\mathrm{du}$ droit commercial. Les ouvriers qui ne peuvent pas rembourser ne remboursent pas, sans autre effet dissuasif qu'une difficulté à retrouver du crédit auprès des mêmes employeurs, en période de mauvaise conjoncture. Certes, le dispositif ne manque pas de donner lieu à des «abus»: des ouvriers utilisent le système pour multiplier les avances sans les rembourser, et des patrons tentent malgré tout d'utiliser les endettements pour retenir leurs ouvriers. Mais les prud'hommes et les justices de paix semblent réprimer avec une certaine efficacité tous ces abus, d'après les enquêtes parlementaires rétrospectives menées en 1848-1850.

En troisième lieu, les tribunaux des prud'hommes et les justices de paix interdisent de porter la moindre appréciation sur la personne ou la conduite des détenteurs de livret : le livret ne doit pas établir autre chose que le contenu demandé par la loi : attestation d'engagement d'ouvrage et, éventuellement, de remboursement différé. Ce point est symboliquement très important car il manifeste un certain maintien de l'esprit révolutionnaire d'égalité civile dans le droit des engagements ouvriers. À l'opposé de normes courantes sous l'Ancien Régime, est consolidée juridiquement la mise hors la loi du paternalisme moral ${ }^{11}$. Des patrons tentent bien de restaurer l'usage d'inscrire des appréciations de bonne ou mauvaise conduite sur les billets de congés, mais tous les prud'hommes étudiés répriment avec rigueur et solennité la moindre inscription dans ce sens : les employeurs n'ont pas le droit de limiter la liberté de ré-engagement de leurs ouvriers, quels que soient leurs motifs de renvoi.

11. Les registres des prud'hommes comportent le plus souvent, en début de fonctionnement, quelques affaires d'appréciations morales sur les livrets ouvriers. Dans tous les cas examinés, sans aucune exception, les inscriptions sont désavouées, et les appréciations négatives donnent lieu à condamnation sévère des patrons, avec remplacement du livret et dommages et intérêts pour les ouvriers. La hiérarchie judiciaire appuie ces jurisprudences, et les autorités administratives qui font imprimer les modèles de livret rappellent également la règle. Sur l'exclusion juridique du paternalisme moral, y compris pendant le Premier Empire et la Restauration, voir l'étude de cas détaillée au début de mon article «Esprit public...", art. cité. 
Un nouvel employeur ne doit pas connaître officiellement les appréciations morales du patron précédent qu'un ouvrier vient de quitter.

\section{Le contraste des jurisprudences française et anglaise (1789-1875)}

Dans le domaine des lois et de la jurisprudence sur le droit de quitter, le contraste entre la France et l'Angleterre, de 1789 à 1875, est donc total : en Angleterre, la tradition de punition pénale des ruptures de contrat (pouvant aller jusqu’à trois mois de prison), lorsque des salariés («servants») quittent irrégulièrement leur employeur («master»), s'accentue et s'étend, au moment même où en France la rupture de contrat ouvrier est dépénalisée radicalement et définitivement par la Révolution. Le contraste est encore plus frappant lorsque l'on examine comment, dans chacun des deux pays, le droit spécifique de l'emploi s'inscrit dans l'ensemble plus général des pénalités pour dettes. En Angleterre, la sanction pénale, et notamment l'emprisonnement pour rupture de contrat, n'était pas très différente de l'emprisonnement pour dette en général, du moins jusqu'au milieu du XIX ${ }^{e}$ siècle ${ }^{12}$. De plus, il semble que pendant plusieurs décennies encore, le droit britannique prolongea une vision morale de la pauvreté comme symptôme de faute, puisqu'il continua de punir les défaillances économiques des plus pauvres ${ }^{13}$. Une telle vision pouvait être encore intériorisée par des délégués ouvriers en 1866, puisque certains d'entre eux, devant la commission parlementaire (Select Committee) sur la loi Master and Servant n'osèrent imaginer ou proposer une suppression complète des sanctions pénales pour dettes impayées ${ }^{14}$.

D'un point de vue comparatif, cette vision morale est d'autant plus frappante qu'elle n'a jamais atteint une telle ampleur en France, où le malheur économique est généralement demeuré digne de respect et de compassion ${ }^{15}$. Durant tout le $\mathrm{xIX}^{\mathrm{e}}$ siècle français fonctionna juridiquement une triple discrimination, mais au profit des plus pauvres : après les flottements de la générosité révolutionnaire, la réorganisation des peines pour dettes sous le Premier Empire réserva les sanctions les plus graves, avec emprisonnement, au seul droit commercial, c'est-à-dire aux seules transactions professionnelles spéculatives, à l'exclusion des transactions professionnelles non spéculatives où furent classées les activités salariées. Les dettes "privées» (par opposition aux dettes «commerciales») furent sanctionnées moins lourdement, suivant un régime de droit commun, et, dans ce contexte, le régime spécial des dettes de travail fit figure de droit d'exception en faveur des ouvriers.

12. Patrick S. Atiyah, The Rise and Fall of Freedom of Contract, Oxford, Oxford University Press, 1979, p. 190.

13. Paul Johnson, "Small debts and economic distress in England and Wales, 1857-1913», dans Economic History Review, 46, 1, 1993, p. 65-87.

14. Parliamentary Papers (House of Commons), 1866 (449), vol. XIII, Dronfield, 799-800, Normansell, 948-952, Williams, 1106, 1152, Odger, 1987.

15. Alain Cottereau, «Providence ou prévoyance? Les «prises en charge» de la santé des ouvriers, au cours des XIxe siècle britannique et français", dans Prévenir, XIX, 2e semestre 1989, p. 21-51. 
La comparaison détaillée du contenu des jurisprudences n'atténue pas le contraste, bien au contraire ${ }^{16}$. On en donnera ici quelques autres exemples. Le témoignage du cordonnier Odger, délégué ouvrier de Londres, nous fournit une configuration intéressante pour la comparaison : il ne se focalise pas sur des abus susceptibles d'être exceptionnels, mais décrit plutôt une difficulté habituelle d'utilisation du droit au bénéfice des ouvriers : ${ }^{17}$

«En ce qui concerne mon métier, je ferai observer que la loi [Masters and Servants, 1823] est presque inapplicable.

- Pour quelle raison? - Pour cette raison que les ruptures de contrat se produisent plus souvent en raison du comportement des maitres que de celui des ouvriers; notre travail, [...] c'est entièrement du travail aux pièces, et fréquemment un employeur négocie pour donner du travail; mais quand nous allons chercher le travail, il ne nous en donne qu'une partie, et nous ne pouvons pas continuer jusqu'au bout. Disons, par exemple, qu'il nous donne le cuir pour faire la semelle, mais pas pour l'empeigne, et l'ouvrier a le travail à faire, et il ne peut avancer. Il y a plusieurs années, il a été jugé dans une affaire dans la City que le contrat commençait quand le travail était donné; mais je n'ai jamais entendu parler d'un cas où un ouvrier assignait un maittre pour une rupture de contrat; c'est toujours pareil, l'ouvrier attend que le maître soit prêt à lui donner l'empeigne, et quand il l'a, il continue le travail. J'ai connu un homme qui attendait parfois neuf ou dix jours, et jusqu'à deux semaines avant d'avoir toutes les choses nécessaires à la poursuite de son travail. Eh bien, bien sûr, dans de telles circonstances, si les ouvriers assignaient en permanence les maîtres, nous aurions une animosité abominable, et tout ce qu'il peut y avoir de mauvais, et les hommes préferent en prendre leur parti, et attendre d'avoir les empeignes, et quand ils les obtiennent, continuer à faire le travail, ou bien aller ailleurs et essayer d'en avoir une paire auprès d'un autre employeur. Cela crée une difficulté, parce

16. La comparaison est cependant limitée par le peu de publications disponibles en Angleterre sur la mise en œuvre effective des Laws of Masters and Servant, sur les textes et contextes des jugements locaux, pour la période étudiée; on a utilisé essentiellement les Parliamentary Papers sur la question, avec leurs listes de cas, (P.P. (H. of C.), 1865 (370), vol. VIII jusqu’à P.P., 1875 (171), vol. LXII, concernant les Contracts of Service ou les Labour Laws) et quelques enquêtes parlementaires donnant occasionnellement des exemples de recours aux tribunaux (P.P., 1824 (51), vol. V, Artisans, Tools and Machinery Exportation... (Joseph Hume)..., P.P., 1825 (414, 437), IV, Combination of workmen..., 1818, I, PP, 1818, Report from Committee on silk..., I, II, III, P.P., H. of Lords, 1823, CLVI, 57, Minutes of evidence... persons employed in the manufacture of silk). L'article de Daphné Simon, "Master and Servant", dans John Saville (ed.), Democracy and the Labour Movement, Londres, Lawrence and Wishart, 1954, p. 160-201, a servi de point de départ synthétique, complété par : John Style, «Embezzlement, industry and the law in England, 1500-1800", dans Maxine Berg, Pat Hudson et Michael Sonenscher (eds), Manufacture in Town and Country Before the Factory, Cambridge, Cambridge University Press, 1983, p. 173-205; David C. WOODS, "The operations of the master and servants act in the Black Country, 1858-1875", dans Midland (s?) History, 7, 1982, p. 93-113; Norma Landau, The Justices of the Peace, 1679-1760, Berkeley, University of California Press, 1984; Robert J. Steinfeld, The Invention of Free Labor. The Employment Relation in English and American Law and Culture, 1350-1870, Chapel Hill, University of Carolina Press, 1991. Voir aussi : Charles Manley Smith, A Treatise on the Law of Master and Servant, Londres, Sweet and Maxwell, 1902.

17. Odger, P.P., réédition de $H$. of C., 1866 (449) Vol. XIII, nº 1809-10. 
que si l'ouvrier essaye d'obtenir une paire d'un autre employeur, alors l'autre employeur peut dire : "Comme tu as l'air d'avoir un trou, je vais te donner du travail en extra». Si, dans ces circonstances, l'ouvrier gardait des pièces du premier employeur plus longtemps que les huit jours autorisés par la loi, il serait responsable des conséquences de la rupture du contrat.

- Alors, quel est le résultat? Des poursuites fréquentes de part et d'autre? Non, il y en a rarement, voire jamais.»

En France au contraire, dans le travail à domicile comme dans le travail en atelier ou en usine, les recours ouvriers, pour non respect du contrat, sont très fréquents (comme sont très fréquents les recours ouvriers en général, dans tous les domaines du «louage d'ouvrage", ainsi qu'on l'expliquera plus loin). Dans le cas des travaux à domicile, des jurisprudences locales permettent aux ouvriers de faire valoir les promesses verbales reçues et sanctionnent par des dommages et intérêts les retards des industriels, de façon plus sévère que les retards ouvriers, estimant les premiers moins excusables que les seconds, dans la plupart des circonstances. Voici un exemple pris dans les soieries lyonnaises, très banal dans la localité, choisi ici pour le contraste frappant qu'il présente, au regard du témoignage de Odger : suivant la jurisprudence habituelle, le temps perdu du fait des négociants est indemnisé, ainsi que les frais de "montage» (c'est-à-dire les frais d'adaptation des métiers aux particularités des pièces de tissus promises, frais que les prud'hommes veillent à faire amortir sur une suite de commandes proportionnée, sous peine de dommages et intérêts). Le compte-rendu de la séance prud'homale qui suit est rédigé par L'Écho de la fabrique, journal de chefs d'atelier qui est en train de s'imposer comme journal de jurisprudence :

«Le Sieur Giraud réclame au Sieur Napoly une somme de 144 fr. pour un grand nombre de journées perdues par le fait de ses employés. Il déclare qu'étant sur le point de régler, parce qu'alors il trouvait de l'ouvrage plus lucratif, on lui promit, s'il voulait continuer l'article, trois pièces de 60 aunes chacune; et nonobstant cette promesse, il n'en a reçu qu'une et on lui refuse les autres.

Le sieur Napoly répond ne vouloir plus occuper cet ouvrier par suite de sa conduite, et déclare ne pas se rappeler de sa promesse de trois pièces, attendu, dit-il, qu'il n'en avait que deux à faire fabriquer et n'en pouvait promettre davantage; mais il reconnait, néanmoins, avoir fait attendre la première pièce très longtemps, et fait perdre plusieurs journées à l'ouvrier depuis cette époque jusqu'à ce jour; il pense les avoir compensées, soit en portant le prix des mouchoirs à $80 \mathrm{c}$. au lieu de $75 \mathrm{c}$., soit par une augmentation de $10 \mathrm{c}$. sur l'avant-dernière pièce, et de $20 \mathrm{c}$. sur la dernière.

Giraud réplique que l'augmentation de $20 \mathrm{c}$. est fausse; qu'il est visible que les chiffres ont été refaits. Jamais, dit-il, je n’ai réclamé plus de 90 c. par mouchoir; cette augmentation, portée sous le titre de bonification, n’a été faite que 
dans l'intention de prouver au conseil que j'avais été satisfait des journées perdues ${ }^{18}$. J'ai payé continuellement mon ouvrier sur le prix de $80 \mathrm{c}$. les premières pièces, et les dernières à $90 \mathrm{c}$.; le prix de la façon n'est donc point pour me défrayer du temps perdu. L'on me refuse de l'ouvrage maintenant, parce que j'ai commis le grand crime, aux yeux de ces messieurs, d'avoir déclaré être payé à 90 c., à un maittre qui fabrique le même article au prix de $80 \mathrm{c}$. Voila le motif pour lequel on me refuse les pièces qu'on m'a promises. Mais je veux poursuivre M. Napoly comme coupable de faux en écriture privée, pour m’avoir refait des chiffres sans mon consentement.

Après une longue délibération le conseil condamne le Sieur Napoly à payer une indemnité de 20 francs au sieur Giraud.»

Ici sont manifestes deux logiques conduisant aux solutions opposées : du côté anglais, une logique de la subordination industrielle fait préjuger sans vérification le bon usage du temps par les chefs manufacturiers. Du côté des prud'hommes français, un souci de l'équité des marchandages individuels conduit à compenser l'inégalité de situation économique par des procédures de réelle réciprocité dans les marchandages, réciprocité qui implique ici la prise en compte du temps perdu occasionné par la gestion des manufacturiers. Cette même dualité de logiques peut expliquer des fonctionnements opposés de la charge de la preuve dans les contestations de promesses verbales. Daphné Simon écrit : «alors que les tribunaux considéraient volontiers que les salaires étaient dus, ils considéraient également que le salaire avait été versé à moins que l'employé puisse démontrer le contraire» ${ }^{19}$, et elle cite un cas, rapporté par une réédition du manuel des juges de paix de Burns, 1837 :

«Un cas jugé quelque temps auparavant au Guildhall était l'action entreprise par l'ouvrier d'une raffinerie sucrière; un témoin prouvait que le plaignant y avait travaillé pendant plus de deux ans, mais Lord Abbott disait qu'il devrait inciter le jury à présumer que les hommes employés de cette façon étaient payés tous les samedis à moins qu'une preuve ne fût apportée de la part du plaignant

18. L'Écho de la fabrique, ${ }^{\circ} 10,10$ mars 1833 , p. 80. L'argumentation rapportée de l'ouvrier concerne une inscription sur son livre de compte (pièce de comptabilité en double, l'une chez le négociant, l'autre aux mains de l'ouvrier chef d'atelier, document légalement obligatoire, distinct du livret). Le négociant a inscrit une bonification après coup sur le livre de compte afin d'éviter de reconnaittre une hausse du prix de façon, c'est-à-dire une hausse du tarif auquel il fait tisser le modèle de tissus en question. C'est une manœuvre destinée à éviter de donner prise à demandes marchandées de hausse de prix. Mais les prud'hommes désavouent la manœuvre en condamnant le manufacturier, en même temps qu'ils accordent l'indemnité de temps perdu demandée. Noter que l'ouvrier chef d'atelier a payé un ouvrier (dit compagnon) pour confectionner la pièce. Comme l'usage sanctionné par les prud'hommes est de toujours accorder une proportion fixe du prix de façon pour salaire des compagnons, et que les compagnons ont l'habitude de vérifier les tarifs de façon sur les livres des chefs d'atelier, le salaire du compagnon sert dès lors ici de preuve du tarif de façon convenu d'avance, et fait confirmer l'accusation de tricherie à l'écriture proférée par le chef d'atelier à l'encontre du négociant.

19. Daphné Simon, «Master and Servant», art. cité, p. 163. 
pour montrer au jury que le plaignant n'avait en fait jamais été payé; et comme une telle preuve ne fut pas apportée, le plaignant fut débouté.»

Aux prud'hommes, les raisonnements et les présomptions sont réglés sur des bases entièrement différentes. Le régime des preuves est dérivé des traditions de droit commercial, interprétées par les tribunaux de commerce. Les manufacturiers et commerçants ont des obligations d'écritures régulières, et ces obligations sont sanctionnées précisément aux tribunaux de commerce par un doute systématique au profit de l'adversaire, lorsque manquent des écritures. La transposition aux prud'hommes s'adapta aux conditions inégales des patrons et des ouvriers par une logique là encore de compensation : seuls les patrons étant tenus à des écritures régulières; les demandes des ouvriers pour défaut de paiement étaient systématiquement satisfaites si les livres de compte des patrons, soumis au besoin à expertise, ne prouvaient pas le contraire.

Le souci d'équité des négociations pouvait ainsi aller jusqu'à des jurisprudences de correction et compensation des inégalités de situation économique. Dans le même esprit avaient été établies des règles autour de la restitution des matières confiées à domicile. Tandis que les statuts et jurisprudences anglaises étaient demeurées répressives, allant parfois jusqu’à instaurer une présomption de vol si les restitutions étaient en retard ou déficientes, la législation française, aussi répressive que le droit anglais avant la Révolution, prit ensuite une voie contraire, après quelques tâtonnements. Sous le Premier Empire des demandes patronales et même prud'homales proposèrent des dispositifs policiers de perquisitions contre le "piquage d'once et vol de matières ", mais les dispositifs préventifs et répressifs furent repoussés au nom de la préservation des libertés civiles. Les prud'hommes établirent alors des jurisprudences sur lesquelles pouvaient s'accorder patrons et ouvriers. Ils allèrent jusqu'à reconnaître aux ouvriers un "droit de rétention", c'est-à-dire un droit de retenir les matériaux confiés, en gages de paiements, si leurs salaires étaient en retard ou impayés. Des audiences prud'homales d'urgence fonctionnèrent alors pour débloquer à l'amiable des refus individuels de poursuivre le travail à domicile.

\section{La disjonction "louage d'ouvrage" et "louage de service" en France, face à l'unification des doctrines anglaises "master and servant"}

En France, les prud'hommes, les justices de paix et les tribunaux de commerce voyaient dans les engagements ouvriers des obligations de résultats, des devoirs de parvenir à un produit matériel suivant les «règles de l'art» (règles de responsabilité issues des compétences techniques). Mais ces obligations n'impliquaient nullement une obéissance à la personne du patron. Seul le «louage de service», terme qui ne s'appliquait pas aux ouvriers, impliquait dans sa nature une soumission aux ordres du maître. En Angleterre, les juris- 
prudences semblent avoir toujours considéré l'emploi des ouvriers comme un engagement à obéir, quelle qu'en soit la justification juridique : coutumes et statuts plus ou moins anciens, puis statuts nouveaux du XIx ${ }^{\mathrm{e}}$ siècle, justifications fonctionnelles de la bonne gestion industrielle et théories du contrat implicite d'obéissance, telle que la formulait par exemple le manuel de Burns en $1837^{20}$ :

«Le salarié s'engage de façon tacite à obéir aux ordres légaux et raisonnables de son employeur dans le cadre des obligations de son contrat."

En France, c'est avant tout l'examen des jurisprudences locales, et non l'examen des doctrines juridiques, ni celui de la jurisprudence nationale, qui permet d'affirmer la disjonction entre louage d'ouvrage et relation de subordination. Cependant, on peut remarquer que ces différences de pratiques correspondent aussi à des différences de doctrines aujourd'hui oubliées, mais présentes sommairement dans la plupart des manuels français de droit civil et commercial, entre 1804 et 1870, reprises aussi parfois en jurisprudence de Cour de cassation. Le vocabulaire technique reprend les termes traditionnels des "prix-fait" (traduction depuis le XvI e siècle de locatio conductio operis) ou «louage d'ouvrage». Les auteurs font correspondre ces contrats de "prix-fait» aux engagements ouvriers marchandés, et plus généralement à tous les engagements de fabriquer une chose déterminée, tandis que les engagements de service sont d'une "autre nature». Les emplois industriels de «journaliers", opposés aux emplois industriels d' "ouvriers" sont assimilables aux relations de «domesticité» ou "service» précisément dans la mesure où ils comportent une mise à la disposition de la volonté du maitre, sans possibilité de discuter et évaluer des tâches à conduire. La distinction juridique «ouvrier/journalier» reprend d'ailleurs une distinction sociale évidente en milieu industriel : les "ouvriers" industriels méprisent les "journaliers» industriels en qui ils voient des "domestiques» ou "valets» soumis aux patrons, estimant tout comme les magistrats que ces «journaliers» ont abdiqué leur indépendance. Statistiquement, les «journaliers» comptent pour environs $10 \%$ des salariés industriels à la fin du Second Empire, et ne doivent pas être confondus avec la catégorie anglaise beaucoup plus large des «non qualifiés».

Leur différence juridique de statut fut tout particulièrement marquée dans le système des preuves en vigueur de 1803 à 1868 : le Code civil, article 1780 , énonçait que «le maître est cru sur son affirmation, pour la quotité des gages; pour le paiement du salaire de l'année échue; et pour les acomptes donnés pour l'année courante». La jurisprudence, sauf de très rares exceptions, considéra que cet article ne s'appliquait qu'aux «domestiques» et «journaliers", à l'exclusion des "ouvriers». On a évoqué plus haut comment 
la tradition des preuves commerciales fit peser sur les seuls employeurs la charge de la preuve du paiement des salaires ouvriers.

Une autre illustration de la dualité entre «louage d'ouvrage» et «subordination", est la longue opposition des prud'hommes aux "règlements d'atelier». Lorsque des patrons affichaient des règlements d'usine et qu'ils prescrivaient une soumission aux ordres de leurs représentants, les prud'hommes récusaient presque toujours la «fiction" contractuelle en vertu de laquelle l'ouvrier entré dans l'atelier était censé connaître le règlement et ne pas l'avoir refusé. Ils s'en tenaient à la légitimité d'une "réalité» contractuelle : ils se chargeaient de vérifier au cas par cas la réalité de l'accord supposé et récusaient toute règle qui ne leur semblait pas équitable, parce que dans le cas de règles inéquitables, il ne pouvait pas y avoir eu accord en toute liberté. De plus, ils donnaient une valeur supérieure et obligatoire aux usages locaux reconnus dans la profession ou le métier.

C'est seulement plus tard, des années 1860 aux années 1880, qu'intervint un changement de jurisprudence, accompagné d'un changement des doctrines juridiques. La hiérarchie judiciaire, puis une partie des patronats et de leurs organisations, menèrent une offensive pour considérer tout engagement ouvrier comme un engagement de soumission aux ordres des industriels. L'offensive vint notamment des compagnies de chemins de fer, bien plus que du secteur manufacturier. En doctrine s'imposa bientôt l'idée que l'engagement ouvrier était un engagement «de service industriel». On établit une équivalence entre «louage de service industriel» et la nouvelle expression "contrat de travail»" 21.

À partir des années 1880, cette doctrine s'imposa dans les jurisprudences, en commençant par le haut de la hiérarchie judiciaire. Elle s'étendit ensuite davantage par voie d'autorité que par conviction, à l'occasion des recours en appel contre les conseils de prud'hommes et les justices de paix. D'un point de vue comparatif, le droit français de l'emploi ouvrier se rapproche alors seulement du droit anglais. Désormais, l'ouvrier français redevient une sorte de "servant», qualité que l'émancipation révolutionnaire avait rejetée avec horreur. En contrepartie des contraintes de soumission, l'ouvrier devient objet de protection législative et support de représentation syndicale légale. En Angleterre, la convergence provient au contraire d'une libéralisation : les sanctions pénales aux ruptures de contrat sont abolies, ce qui achève l'évolution vers une justification purement contractuelle des relations de "master and servant» ${ }^{22}$.

21. Plusieurs aspects de ce changement sont retracés dans mes articles, notamment, en ce qui concerne les règlements d'atelier, dans "Justice et injustice ordinaire... ", art. cité, p. 55-58. La première occurrence de l'expression «contrat de travail» que j'ai relevée remonte à 1886.

22. Un malentendu comparatif profond a été répandu à partir d'une mythologie établie en France sous la Troisième République : des juristes républicains du travail ont voulu croire et ont fait croire que le «louage de service» était une catégorie du Code civil qui s'appliquait dès l'origine à l'emploi des ouvriers. Les considérations comparatives de Kahn-Freund, par exemple (Otto Kahn Freund, «Blacks- 


\section{Le sens de la justice des ouvriers, et son traitement institutionnel comparé en France et en Grande-Bretagne}

Pour approfondir la comparaison, une question plus large s'impose : quels rapports s'établissaient entre le sens de la justice, la légitimité et les systèmes de droit? Une clé comparative a déjà été suggérée en introduction : l'institution prud'homale a pu consacrer et faire jouer collectivement des jugements partagés de justice, entre élus patrons et élus ouvriers, sans prendre le chemin habituel de la représentation publique. Le contraste est particulièrement sensible avec les trade-unions et leur légitimation dans la société anglaise du XIX siècle.

Si nous prenons pour point de départ les années 1820 , celles où, en Grande-Bretagne ont été établies simultanément les extensions punitive des lois Master and Servant, les suppressions des dernières régulations statutaires du travail, et la libéralisation du droit des trade-unions, nous arrivons à un schéma de symétrie inverse parfaite entre la négociation face-à-face et la négociation collective par représentants publics : en France est prohibée et punie pénalement la négociation collective par délégation publique, tandis qu'est protégée, encouragée et régulée judiciairement au profit des ouvriers la négociation face-à-face. En Grande-Bretagne, la négociation face-à-face, déjà plus faible stratégiquement qu'en France, dans les conditions démographiques et économiques de l'industrialisation anglaise, subit une intervention juridique ouverte inégale au profit des employeurs, tandis des corrections collectives, pratiquées traditionnellement en dialectique avec le droit et les magistrats locaux, se voient encouragées par la loi au nom d'un libéralisme qui «laisse passer» la représentation collective «volontaire».

Pour comprendre l'ampleur du contraste, il importe d'abord de ne pas sous-estimer la réalité et le succès institutionnel des recours aux prud'hommes ${ }^{23}$ : à la différence des recours judiciaires habituels, l'appel aux prud'hommes n'est ni une déclaration d'hostilité ni un transfert dans un monde lointain et supérieur de légalité, susceptible de rendre un "arbitrage». Ce n'est pas non plus le système paternaliste des justices de paix anglaises, (bien que des rapprochements soient possibles, dans les meilleurs des cas, avec des juges de

tone's neglected child : the contract of employment ", The Law Quarterly Review, volume 93, octobre 1977, p. 508-528), sont induites en erreur par les auteurs français auxquels il a fait confiance. Les usages post-révolutionnaires de Pothier sont plus précis qu'il ne croit, et modifient sciemment une distinction entre "louage d'ouvrage» et "louage de service» effectuée par Pothier lui-même dans ses traités du louage d'ouvrage puis du louage maritime : Robert-J. Pothier, Traité du contrat de louage, selon les règles tant du for de la conscience que du for extérieur, Paris, Debure aîné, 1764; Traité des contrats de louage maritime, Paris, Debure aîné, 1769.

23. Pour plus de détails sur le succès des prud'hommes, voir Alain Cottereau, "Justice et injustice ordinaire...», art. cité. Il resterait à situer et comparer le succès des justices de paix françaises par rapport aux prud'hommes, tâche qui n’a pas encore été effectuée dans l'historiographie française. Mes sondages en archives de justices de paix laissent supposer une beaucoup plus grande variété des pratiques, avec des cas de figure plus paternalistes, d'autres cas plus répressifs, et enfin de nombreux cas alignés sur les jurisprudences prud'homales, au nom de l'équité et de la protection des faibles. 
paix populaires, réputés particulièrement équitables ${ }^{24}$ ). À la différence des justices corporatives et municipales françaises d'Ancien Régime, l'institution prud'homale reposait sur la «conciliation" et non sur l'"arbitrage». Entre les deux, l'écart de philosophie est radical : l'arbitrage était compatible avec une «justice paternelle» qui donnait aux notables un pouvoir arbitraire d'apaisement au nom de la supériorité de leurs lumières, du haut de leur position sociale; par contre la "conciliation» supposait que les parties en désaccord étaient invitées par les "conseillers" prud'hommes à réévaluer elles-mêmes leur situation jusqu'à trouver une solution acceptable par tous les points de vue en présence. Elle présupposait une «capacité de juger» des ouvriers comme des patrons, sous les conditions institutionnelles organisées en conséquence : les prud'hommes n'étaient pas exactement "à majorité patronale» jusqu'en 1848, comme il a été trop souvent écrit. Ils relevaient d'une sorte de suffrage censitaire interne aux milieux ouvriers comme aux milieux manufacturiers. Mais surtout, ils fonctionnaient presque toujours au «consensus de justice», non seulement entre conseillers, mais aussi entre justiciables. Un "consensus» toujours visé, souvent atteint. Ce que symbolisait et organisait à la fois les «bureaux particuliers» de conciliation, sortes de première instance où se réglaient 80 à $100 \%$ des litiges enregistrés : ils étaient obligatoirement composés d'un patron et d'un ouvrier, alors que les organes de conciliation d'Ancien Régime, beaucoup moins sollicités et beaucoup plus controversés, ne comportaient le plus souvent que des «maittres».

Le système des conciliations face-à-face, dites à tort «individuelles", avait une portée collective essentielle. Il entretenait un débat permanent interne aux milieux professionnels, et donnait lieu à de véritables systèmes de règles non écrites, gérées au jour le jour sous la surveillance de tout le milieu concerné. Prenons l'exemple des «tarifs de façon" lyonnais, à peu près équivalents aux "piece-rates». Les prud'hommes lyonnais établissent dès leurs premières réunions (janvier 1807) un tarif de prix, imprimé comme les « lists» du régime des Spitalfields Acts. Les listes sont périodiquement remises à jour jusqu'en 1831, époque où une nouvelle fixation négociée subit un désaveu gouvernemental: les Lyonnais se voient dire, sur plainte d'une partie des patrons, que les nouveaux tarifs ne peuvent avoir de valeur obligatoire, mais seulement une valeur morale. Ce désaveu déclencha la fameuse première révolte des canuts. Ici appa-

24. En prenant notamment pour références C. R. Dobson, Masters and Journeymen. A Prehistory of Industrial Relations, 1717-1800, Londres, Croom Helm, 1980 et Norma Landau, The Justices of the Peace..., ouv. cité. La monographie de Gail Malmgreen, Silk Town : Industry and Culture in Macclesfield, 1750-1835, Hull, Hull University Press, 1985, est la seule qui m'a permis de faire des comparaisons entre archives prud'homales des soieries et jugements de Local Courts dans la même branche anglaise à la même époque. Les brèves indications qu’elle donne (notamment p. 40-41), à partir du notebook d'un magistrat local réputé pour son "fairness", montrent que les causes de litige et les arguments de justice étaient proches, que l'effectif des recours par rapport à la population était assez important, tout en restant loin des niveaux de recours français aux prud'hommes. Il y avait à peu près un recours ouvrier contre deux recours patronaux, ce qui semble une proportion forte et inattendue pour l'Angleterre, alors qu'en France les recours ouvriers étaient généralement beaucoup plus nombreux que les recours patronaux. 
raît une particularité essentielle, par delà de multiples analogies avec les «lists» négociées des trade-unions: en Angleterre à la même époque, on constate que "la puissance morale» d'un accord collectif, avec l'appui actif des Unions, pouvait se manifester, dans les cas les moins conflictuels, par un respect effectif des règles négociées, sans appui judiciaire ni étatique.

À Lyon, comme dans toute la France, le respect des règles ne pouvait être envisagé de cette manière. Un nouveau régime s'y stabilise à partir de 1832, typique du mode de fonctionnement des régulations prud'homales du pays jusque dans les années 1860. En dépit du retrait du tarif officiel, les canuts obtinrent en partie gain de cause. S'il n'y eut pas de liste des prix obligatoire, fonctionna bien un système officieux de prix de références, appelé "le cours", que les prud'hommes appuyèrent et contribuèrent à faire respecter. En conciliation, et même en jugement, ils prononçaient une hausse de salaire lorsque le "prix convenu » était jugé insuffisant. Ceci en contradiction flagrante avec le libéralisme officiel et les doctrines juridiques civilistes (mais non en contradiction avec le droit commercial). Les notables lyonnais, comme les manufacturiers de nombreuses cités françaises, assumaient un double langage. Le bon fonctionnement des relations industrielles avait ses règles locales, que, pour des raisons d'efficacité, il importait de ne pas exposer à «l'opinion publique" (c'est-à-dire la scène publique nationale de l'époque). C'était une sorte de régime de clandestinité, qui ne pouvait tenir que dans la mesure où les notables patronaux obtenaient une crédibilité et une discipline suffisante de leurs pairs pour éviter les appels à la hiérarchie des tribunaux.

S'il fallait traduire aujourd'hui par une expression anglaise ancienne les pratiques prud'homales consensuelles, ce serait en inversant un leitmotiv des artisans protestataires de l'époque contre la concurrence sans principes ("unprincipled») : les régulations prud'homales concouraient à une concurrence avec principes ("principled»). Peut-être serait-il aussi pertinent d'y voir une parenté avec la "moral economy" de Edward P. Thompson, revisitée en $1991^{25}$, pour peu que cette notion soit débarrassée de ses réductions trop classistes et élargie à une dialectique plus complexe de la reconnaissance de légitimité. On peut alors considérer les régulations prud'homales comme un autre cas historique où l'économie politique et l'équité s'interpénètrent. Une compénétration incompréhensible au regard de la théorie économique classique, pour des raisons analogues à l'opacité de la "moral economy».

En Grande-Bretagne, durant les années 1820-1870, les zones d'initiative pour prévenir la concurrence sans principes, pour la dénoncer ou la corriger, relevaient d'une topologie de la "public sphere» très différente de celle de la sphère publique française ${ }^{26}$. Au-delà de ce que les Webb appelaient, de

25. Edward P. Thompson, Customs in Common, Londres, Merlin Press, 1991.

26. Le terme de public sphere s'inspire à la fois de la tradition habermassienne : Jürgen Habermas, "Vorwort zur Neuauflage», $17^{\mathrm{e}}$ édition de Strukturwandel der Öffentlichkeit, Frankfurt-a-M., Suhrkamp, 
façon trop managériale et évolutionniste, dans Industrial democracy ${ }^{27}$, les différentes méthodes (de promulgation, de négociation collective, etc) se jouait peut-être, plus fondamentalement, un autre régime du sens de la justice, de la publicité, et du pouvoir d'intervention, commun à l'ensemble de la vie politique britannique. Pour un lecteur français, un symptôme frappant de cet autre régime est l'usage fait par les ouvriers anglais de termes tels que "legal (illegal) men, lawful men, fair men" (hommes en accord avec la loi, hommes en règle, hommes équitables), à propos des ouvriers qui respectent ou non des règles (rules) établies par les Unions ${ }^{28}$. Des règles établies parfois par négociation collective, mais aussi des règles établies unilatéralement, en opposition et en guerre déclarée contre la légalité étatique, judiciaire et (ou) patronale. Dans ce dernier cas, les termes de "legal (illegal) men, lawful men" seraient intraduisibles, incompréhensibles en France, à l'époque ${ }^{29}$.

Dans les situations les plus voisines, lorsque des collectifs ouvriers français imposent clandestinement des règles de négociation et de conditions de travail, ils ne prétendent pas à une légalité autonome. D'une part, sauf circonstances très exceptionnelles de conflit ouvert et organisé, ils ne disposent pas de procédures d'assemblées et de délibération suffisamment ouvertes (clandestinement ou publiquement) pour prétendre ensuite imposer des obligations au nom d'un intérêt collectif dûment établi. D'autre part, lorsqu'il y a conviction partagée de justice de la cause, s'ouvre une recherche de reconnaissance auprès des magistrats locaux et des patrons les plus accessibles. C'est une tactique qui semble souvent couronnée de succès ${ }^{30}$. Lorsqu'il y a échec, le milieu professionnel ouvrier concerné ne se subdivise pas pour autant en «legal men» (ou "legal shop», atelier en accord avec la loi) et «illegal

1990, p. 11-50, et de la tradition arendtienne : Hannah Arendt, The Human Condition, Chicago, University of Chicago Press, 1958.

27. Sidney et Beatrice Webb, Industrial Democracy, Londres, Longmans, Green and co, 1897.

28. Occurences de termes relevées dans P.P. 1824 (51), Vol. V, Artisans,... et dans Henry Mayhew, The Unknown Mayhew: Selections from the Morning Chronicle, 1849-1850, textes édités par Edward P. Thompson et Eileen Yeo, Londres, Merlin Press, 1971.

29. L'incompréhensibilité n'est valable que progressivement, à partir des années 1820. Auparavant, sous l'Ancien Régime et au début de la Révolution, les assemblées ouvrières françaises comportaient elles aussi leur appareil législatif, et utilisaient un vocabulaire commun avec les procédures nationales de délibération judiciaire, législative ou gouvernementale. On en retrouve certes des exemples de prolongements durant la première moitié du XIX ${ }^{\mathrm{e}}$ siècle, mais ils se font de plus en plus rares.

30. La réalité de ces succès n'est pas facile à établir, dans l'état actuel de l'historiographie. Il faut des enquêtes qui puissent retracer la vie normative des milieux professionnels, les légitimations et illégitimations de règles dans différentes conjonctures, ce qui exige de dépasser les limites des approches héroïques, policières ou gestionnaires des conflits professionnels. Les archives policières ou judiciaires qui, elles, rendent compte en général des échecs, et des affrontements violents, portent cependant aussi des traces de ces recherches de légitimations, si l'on veut bien être attentif aux déceptions normatives qui s'y expriment et en tirer les conséquences sur la prévalence des normes qui donnent lieu à déception. Souvent les enquêteurs rapportent que les délégués ouvriers arrêtés à l'occasion de conflits sont stupéfaits d'être traités comme des délinquants, alors qưils menaient un combat moral pour la justice des conditions de travail. Que ces déceptions soient sincères, ou que l'historien les soupçonne d'être simulées pour la défense des intéressés, ne change pas la conclusion que l'on peut en tirer ici sur la polarisation normative de l'espace où ils se situent: l'opportunité tactique présuppose une vraisemblance et une recevabilité des attitudes morales qu'il est judicieux d'afficher. 
men». Sémantiquement, il n'y a pas deux légalités. Il y a d'une part une légitimité, une conviction de justice sur les principes à faire respecter. Il y a d'autre part un milieu professionnel, patrons et ouvriers, qu'il faut convaincre, qu'il faut améliorer, en faisant appel au besoin à «la» loi, en redressant s'il le faut ses mauvaises interprétations et en modifiant ses énoncés qui se prêteraient à des usages injustes.

En d'autres termes, les procédés et les zones où se joue la distance entre légitimité et légalité ne sont pas les mêmes. En Grande-Bretagne, à l'occasion de la crise chartiste, des premiers élargissements électoraux et des errements de l'appareil judiciaire, la «Law» devient plus distante du citoyen, et l'État capte davantage d'attentes et de déceptions. Si le «self help» redistribue les irresponsabilités collectives, les radicalismes ouvriers, de leur côté, hissent en débat national et en enjeu étatique la recherche des réponses aux injustices de l'industrialisation. Dans ce contexte, l'exaspération universaliste et la sensibilité aux discriminations de classe vont de pair. Mais, dans le cas britannique, la tension entre légitimité universaliste et particularismes de classe ne s'arrête pas à la vie symbolique des justifications, et c'est là une grande différence avec la France de la même époque : des lieux de légitimité incompatibles s'organisent, tant en logiques ouvrières qu'en logiques de classes dominantes. Les discriminations du droit "Master and Servant» et les "legalities» ouvrières hors reconnaissance nationale forment couple et se renforcent dans l'hostilité mutuelle.

Par contraste, les régulations prud'homales, des années 1800 aux années 1860 représentent une autre manière de traiter la tension entre légitimité et légalité, entre justice universaliste et particularités de classe. Ici, personne n’a renoncé à la reconnaissance universelle du «bon droit» : ni les ouvriers à l'égard des patrons et des autorités, ni les autorités et les patrons à l'égard des ouvriers. L'expérience historique des prud'hommes traduit la façon dont la sphère publique fut instaurée en France sous la Révolution puis fut bridée sans être brisée sous les régimes successifs du Premier au Second Empire. La peur panique des foules, de la démagogie et des manifestations publiques a fait interdire ou limiter les libertés de presse et de réunion, comme chacun le sait. Mais en contrepartie des verrouillages d'un espace public national, se sont développés des publics locaux. Non pas simplement des sphères d'expression d'opinion, mais des sphères de délibération : les autorités locales, administratives et judiciaires y demeuraient sous contrôle des citoyens locaux; elles étaient obligées de faire reconnaître la validité de leurs actes par une multitude de contraintes morales, quels que soient leurs modes de désignation. L'invention des «common law» prud'homales a constitué, dans le domaine du droit, une réponse originale à ces exigences.

Alain Cottereau est directeur d'études à l'École des Hautes Études en Sciences Sociales, Centre d'Étude des Mouvements Sociaux 